HNO 2019 $\cdot 67: 561-562$

https://doi.org/10.1007/s00106-019-0695-6

Online publiziert: 7. Juni 2019

๑ Springer Medizin Verlag GmbH, ein Teil von Springer Nature 2019

B. A. Stuck' $\cdot$ A. Beule 2 D. Jobst ${ }^{3} \cdot$ L. Klimek ${ }^{4} \cdot$ M. Laudien ${ }^{5} \cdot$ M. Lell ${ }^{6} \cdot$ T. J. Vogl ${ }^{7} \cdot$ U. Popert ${ }^{8}$

${ }^{1}$ Klinik für Hals-, Nasen- und Ohrenheilkunde, Universitätsklinikum Gießen und Marburg GmbH, Standort Marburg, Marburg, Deutschland; ${ }^{2}$ Klinik für Hals-, Nasen- und Ohrenheilkunde, Universitätsklinikum Münster, Münster, Deutschland; ${ }^{3}$ Institut für Hausarztmedizin, Universitätsklinikum Bonn, Bonn, Deutschland; ${ }^{4}$ Allergiezentrum Wiesbaden, Wiesbaden, Deutschland; ${ }^{5}$ Klinik für Hals-, Nasen-, Ohrenheilkunde, Kopf- und Halschirurgie, Universitätsklinikum Schleswig-Holstein, Campus Kiel, Kiel, Deutschland; ' ${ }^{6}$ Institut für Radiologie und Nuklearmedizin, Klinikum Nürnberg, Nürnberg, Deutschland; ${ }^{7}$ Institut für Diagnostische und Interventionelle Radiologie, Uniklinik Frankfurt, Frankfurt am Main, Deutschland; ${ }^{8}$ Praxis für Allgemeinmedizin, Kassel, Deutschland

\title{
Erratum zu: Leitlinie
} "Rhinosinusitis" - Langfassung

\section{S2k-Leitlinie der Deutschen Gesellschaft für Allgemeinmedizin und Familienmedizin und der Deutschen Gesellschaft für Hals-Nasen- Ohren-Heilkunde, Kopf- und Hals- Chirurgie e.V.}

\section{Erratum zu:}

HNO 2018 66:38-74

https://doi.org/10.1007/s00106-0170401-5

Die Autoren weisen auf folgendes hin:

In der Langfassung der o.g. Leitlinie ist uns ein Fehler unterlaufen. Hier wurde "Carboxymethylcellulose“ irrtümlich statt „Carboxymethylcystein (Carbocystein)" angegeben. Richtig muss es daher heißen:

Auf Seite 60 (mittlere Spalte, 3. Absatz):

Die Wirksamkeit von Clarithromycin ( $200 \mathrm{mg} / \mathrm{die})$ ließ sich bei Erwachsenen durch gleichzeitige Einnahme von Carboxymethylcystein (Carbocystein) (1500 mg/die) noch steigern: sowohl die Rate der Ansprecher (64,2 vs. 45,6\%), wie auch das Ausmaß der anterioren und posterioren nasalen Sekretion besserte sich mehr, als bei der alleinigen Einnahme von Clarithromycin bei einer großen eingeschlossenen Patientenanzahl $(N=425)$ [130].

Sowie auf S. 60 weiter unten:

Auch für die Symptome Schleimhautschwellung (75,9\% vs. $66,0 \%)$, die
Menge des Nasensekrets $(72,3 \%$ vs. $62,7 \%)$, die Art des Nasensekrets (91,9\% vs. $86,6 \%$ ) und das post-nasale Sekret ( $86,2 \%$ vs. $70,3 \%$ ) zeigte sich eine höhere Besserungsrate für die Kombination von Clarithromycin mit Carboxymethylcystein (Carbocystein).

Die Kurzfassung, die Miniaturversion und der Algorithmus der o.g. Leitlinie sind nicht betroffen.

\section{Korrespondenzadresse}

Prof. Dr. med. B. A. Stuck

Klinik für Hals-, Nasen- und Ohrenheilkunde, Universitätsklinikum Gießen und Marburg $\mathrm{GmbH}$, Standort Marburg

Baldingerstraße, 35043 Marburg, Deutschland boris.stuck@uk-gm.de

Die Online-Version des Originalartikels ist unter https://doi.org/10.1007/s00106-017-0401-5 zu finden. 
Hier steht eine Anzeige.

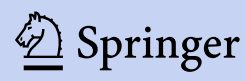

